\title{
Aneurysm of a Paraumbilical Collateral Vein
}

\author{
Karen J. Stuck, MD, Jonathan M. Rubin, MD, PhD, Barry Gubin, MD
}

Sonography and computed tomography have both been shown to be useful in the detection and definition of venous collaterals in portal hypertension. ${ }^{1=4}$ The paraumbilical veins are one of the more common collateral pathways demonstrated. Increasing size of this collateral pathway has been positively correlated with the development of gastroesophageal varices and the degree of extrahepatic shunting. ${ }^{5}$ Focal dilatations of collateral vessels are uncommon, usually described in the portal vein. ${ }^{3}$ We report a case of a paraumbilical vein aneurysm imaged with CT and color flow and gray-scale Doppler sonography.

\section{CASE REPORT}

A 63-year-old man with a long history of alcohol abuse, cirrhosis and known esophageal varices presented to an outside hospital for recurrent upper gastrointestinal bleeding. As part of his inpatient evaluation, an abdominal sonogram was performed, which identified a "cystic structure in the left abdomen anteriorly which probably represents a pancreatic cyst." He was referred to the Ann Arbor VA Medical Center for a computed tomography (CT) examination. Changes consistent with portal hypertension were seen, including enlargement of a paraumbilical vein and numerous superficial periumbilical collateral vessels resulting in a caput medusae (Fig. 1). Approximately $8 \mathrm{~cm}$ above the umbilicus there was a focal aneurysm measuring $5 \mathrm{~cm}$ in AP diameter. Thrombus was identified in the anterior lumen. A color-flow and gray scale Doppler examination performed at the University of Michigan Hospitals showed characteristic venous flow in the vein, and confirmed thrombus within the aneurysm (Fig. 2A). Multiple superficial collaterals were also identified (Fig. 2B). The aneurysm accounted for the "cystic structure" imaged on the outside sonogram. The patient's esophageal varices were treated

Received March 21, 1988, from the Department of Radiology, University of Michigan Medical School, Ann Arbor, Michigan. Revised manuscript accepted for publication April 25, 1988.

Address correspondence and reprint requests to Dr. Stuck: Ann Arbor VA Medical center (114), 2215 Fuller Road, Ann Arbor, Michigan 48105. with sclerotherapy. No further evaluation or intervention of the aneurysm has been performed at this time.

\section{DISCUSSION}

The vessel in the falciform ligament demonstrated in portal hypertension is frequently referred to as a recanalized umbilical vein. Normally, shortly after birth, the umbilical vein atrophies within the falciform ligament and forms the ligamentum teres. Lafortune et $\mathrm{al}^{6}$ showed that the falciform ligament contains one to three tiny paraumbilical veins, and that these are the vessels that increase in both size and number in portal hypertension. The umbilical vein remnant may remain patent in its hepatic portion for a short distance, thus accounting for the small vessel $(<3 \mathrm{~mm}$ ) that can occasionally be depicted near the left portal vein in normal subjects. However, in their study, the ligamentum teres close to the umbilicus was not patent due to a reopened umbilical vein.

The radiographic evaluation of patients with portal hypertension continues to change. Computed tomography has become an important complement to angiographic evaluation, particularly in the preoperative workup of patients for distal splenorenal shunt. The CT increased the detection of paraumbilical and retroperitoneal varices, while angiography is better for imaging peripancreatic collaterals and cavernous transformation." Presence and direction of flow within the portal circulation and collateral vessels can also be studied accurately with duplex Doppler sonography. ${ }^{78}$

Although our case does not present new information on the imaging of patients with portal hypertension, the aneurysmal dilatation of a collateral vessel is unusual. Color-flow imaging of the collateral circulation clearly shows the superficial periumbilical collaterals as well as the thrombus within the aneurysm. The prognosis for the patient is uncertain. He has had repeated episodes of ascites as well as esophageal variceal bleeding requiring treatment by sclerotherapy. Given the degree of extrahepatic shunting, the surgeons have not been anxious to operate on the aneurysm. 


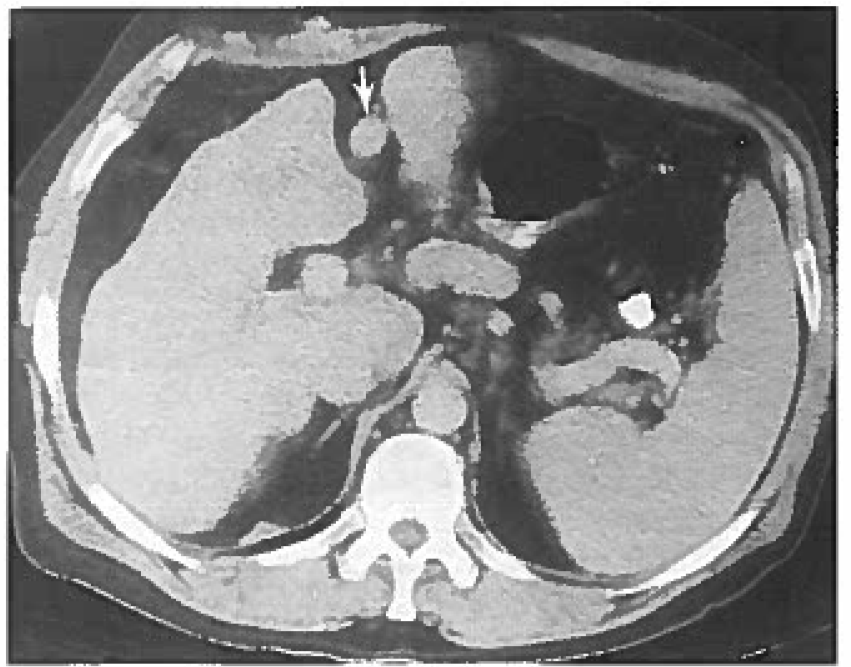

A

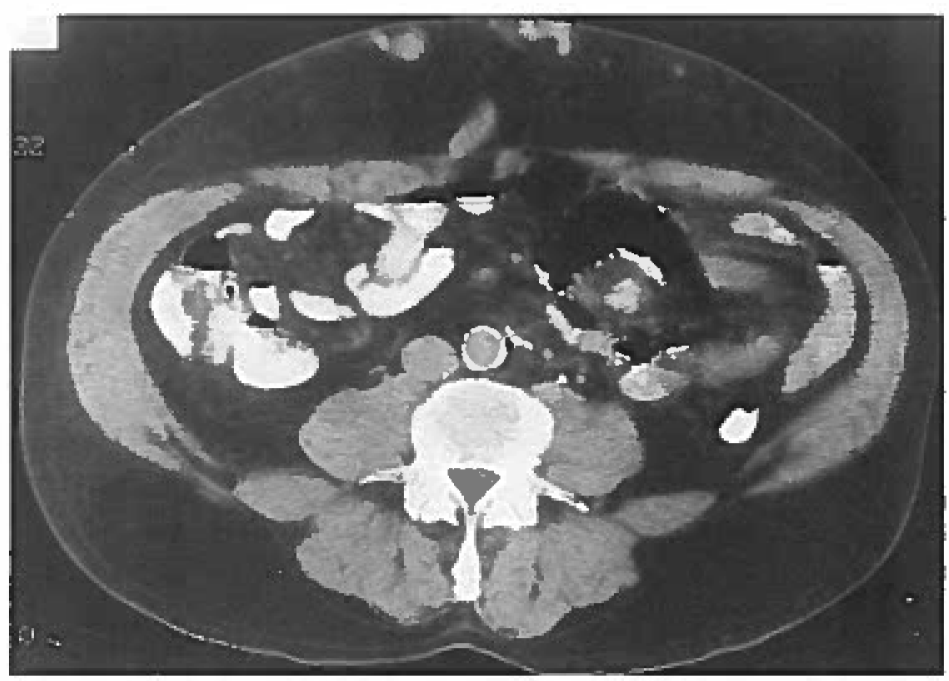

C

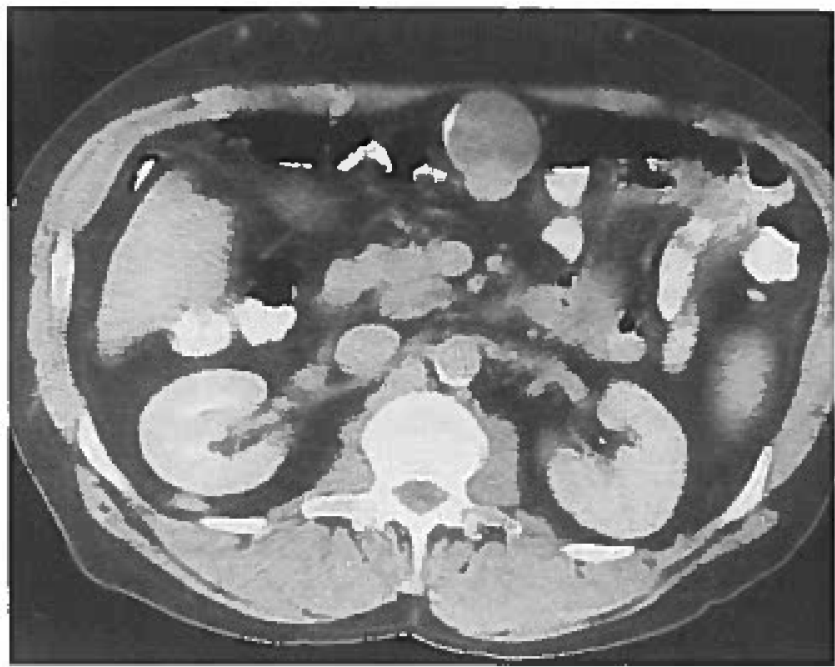

B

Figure 1 A, Changes of portal hypertension and cirthosis are seen on the CT scan, including irregular hepatic contour with an enlarged caudate lobe, splenomegaly, and a large paraumbilical vein within the falciform ligament (arrow) $, \mathrm{B}, 8 \mathrm{~cm}$ caudal to $\mathrm{A}$, there is aneurysmal dilatation of the collateral vessel. Low attenuation thrombus is seen in the anterior lumen. The aneurysm extended for $6 \mathrm{~cm}$. C, At the umbilicus, superficial periumbilical veins are seen, the caput medusae. 


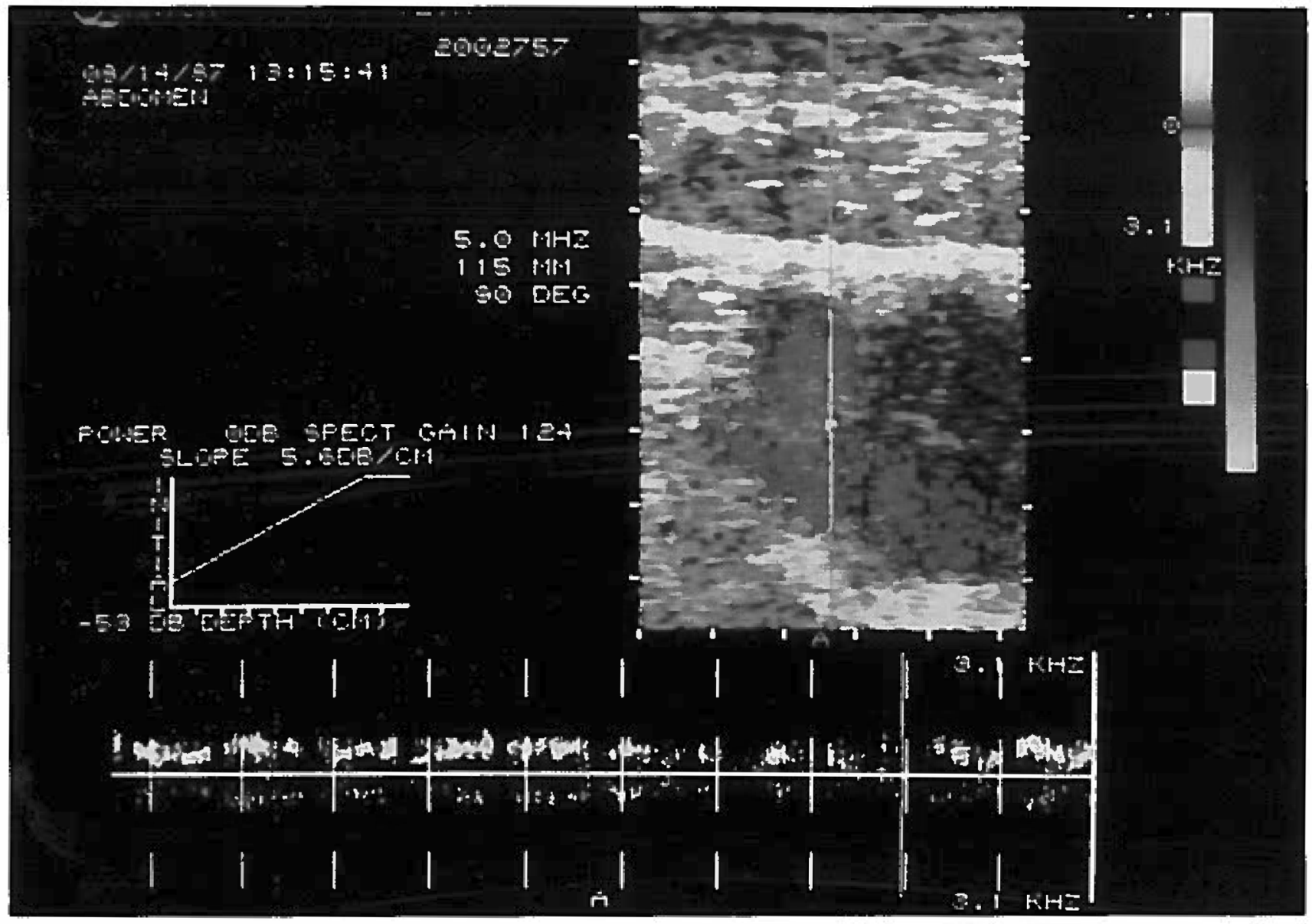

A

Figure 2 A, The Doppler waveform shows characteristic venous flow within the lumen of the aneurysm. Thrombus (blue) is noted anteriorly in the color-flow image. B, Transverse color-flow image near the umbilicus shows the superficial collateral vessels (red) in the subcutaneous tissues. 


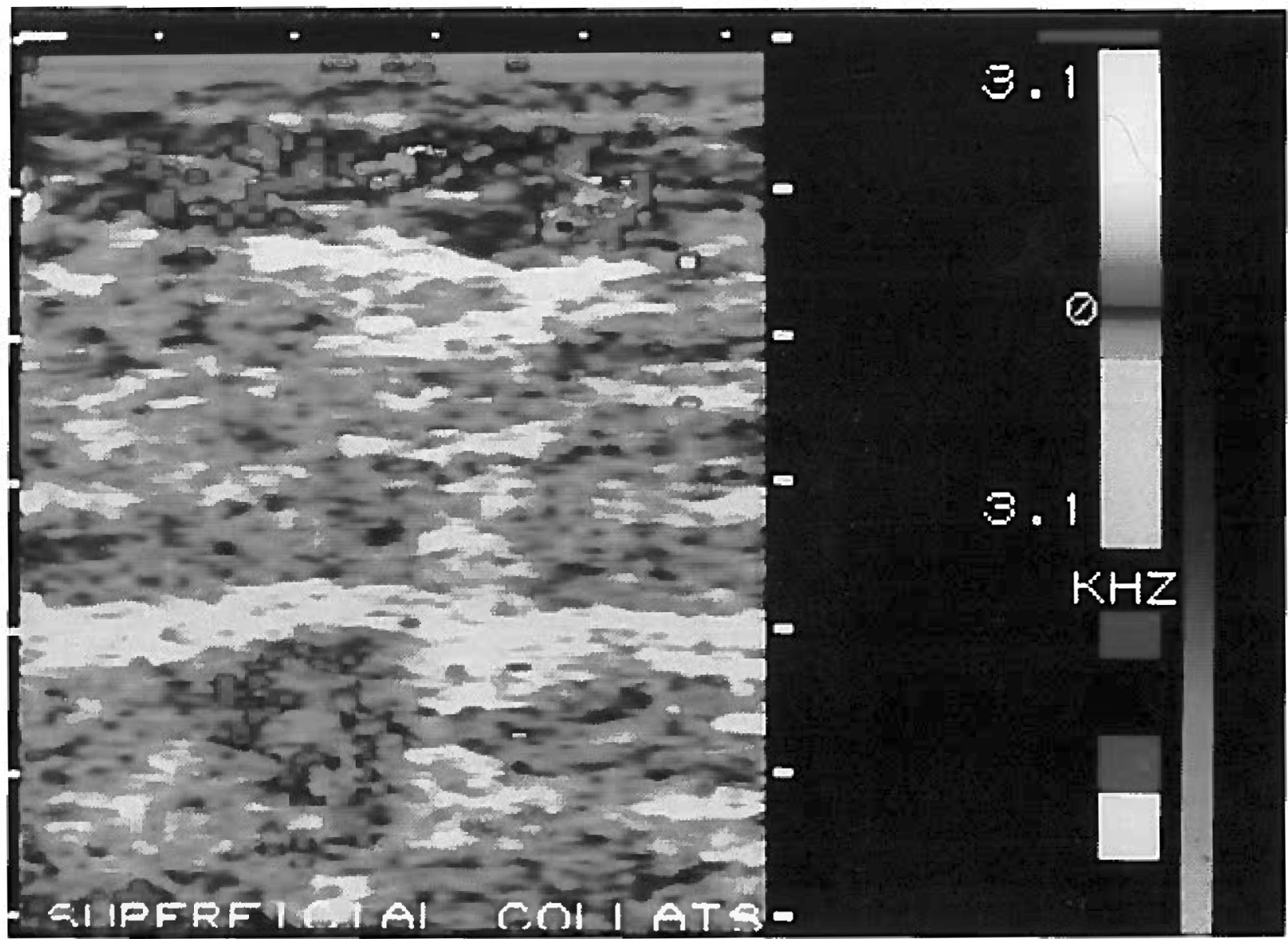

B

\section{REFERENCES}

1. Foley WD, Varma RR, Lawson TL, et al: Dynamic computed tomography and duplex ultrasonography: Adjuncts to arterial portography. J Comput Assist Tomogr 7:77, 1983

2. DiCandio G, Campatelli A, Mosca F, et al: Ultrasound detection of unusual spontaneous portosystemic shunts associated with uncomplicated portal hypertension. J Ultrasound Med 4:297, 1985

3. Ishikawa $T$, Tsukune $Y$, Ohyama $Y$, et alt Venous abnormalities in portal hypertension demonstrated by CT. AJR 271, 1980

4. McCain AH, Bernardino ME, Sones PJ Jr, et al: Varices from portal hypertension: Correlation of CT and angiography. Radiology 154:63, 1985

5. Aagaard J, Jensen LI, Sorensen TLA, et al: Recanalized umbilical vein in portal hypertension. AJR 139:1107, 1982

6. Lafortune $M$, Constantin A, Breton $G$, et al: The recanalized umbilical vein in protal hypertension: A myth. AJR 144:549, 1985

7. Patriquin H, Lafortune M, Burns PN, et al: Duplex Doppler examination in portal hypertension: Technique and anatomy. AJR 149:71, 1987

8. Nelson RC, Lovett KE, Chezmar JL, et al: Comparison of pulsed Doppler sonography and angiography in patients with portal hypertension. AJR 149:77, 1987 